NEUE

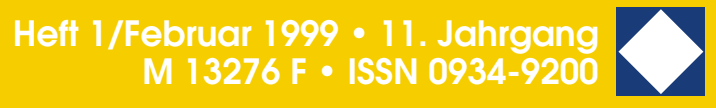

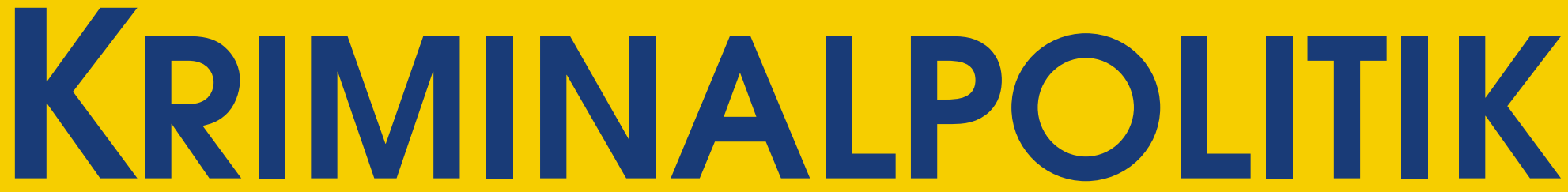

Forum für Proxis, Pollitik und Wissenschofit

DOKUMENIATION:

Soziale Trainingskurse und andere neve ambulante Maßnahmen

INTERVIEW:

Kriminalpolitisches Gespräch mit Christion Pfeiffer über Jugendkriminalitöt als Folge sozialer Unterpriviligierung.
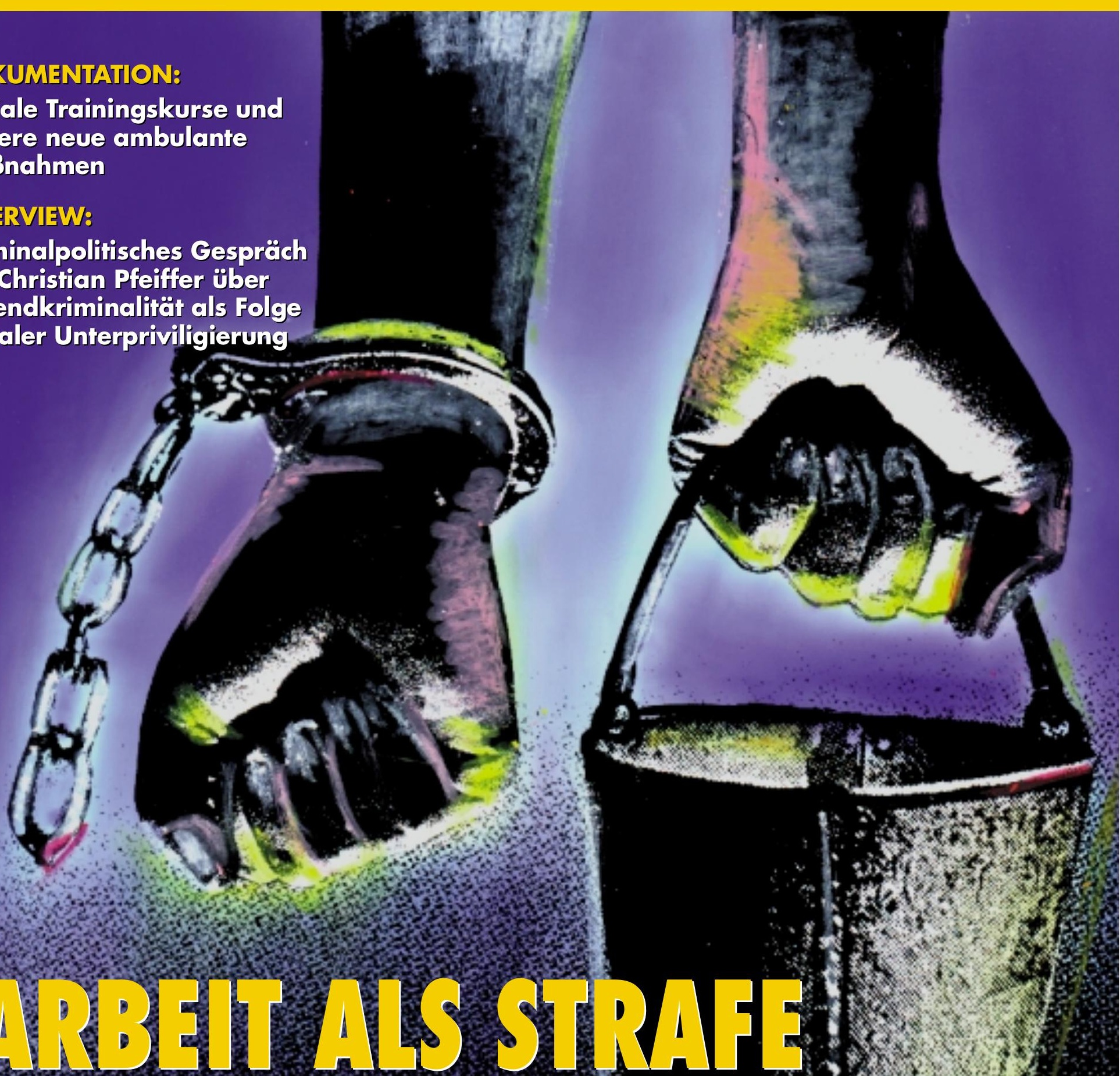

120ie Cemeinitizige Arbeit wird Hauptstrafe, doch noch gibt es viele offene Fragenu

.

3

3 


\section{Alternative Alternativen}

Die Gefängnisse sind voll. Also muß man neue bauen. So einfach ist das noch immer, folgt man der populistischen Logik der verantwortlichen Justizminister jedweder politischen Couleur. An Alternativen zur Freiheitsstrafe besteht freilich kein Mangel. Zum Beispiel soziale Trainingskurse, Tatausgleich oder gemeinnützige Arbeit. Von Entkriminalisierung sowie Ersaztfreiheisstrafen- und U-HaftVermeidung gar nicht zu reden. In der NEUEN KRIMINALPOLITIK werden diese Alternativen bekanntlich eher wohlwollend kommentiert bis aktiv gefordert. Aber nicht jede Alternative ist eine gute Alternative. Insbesondere wenn vom elektronisch überwachten Hausarrest und von Fahrverboten die Rede ist, stellen sich kriminalpolitische Bedenken ein. Problematisch daran ist die Ausweitung des Sanktionsapparates auf immer mehr Lebensbereiche, auf den Privathaushalt und die Nutzung des öffentlichen Raums.

Erfreulich ist, daß Modelle und Projekte zur Freiheitsstrafenvermeidung in der öffentlichen Debatte verstärkt Aufmerksamkeit erfahren. Es steht aber zu befürchten, daß dabei die falschen Alternativen den Vorzug erhalten. Bei allen Reformvorhaben wäre zudem strikt darauf zu achten, daß sie ausschließlich zur Vermeidung einer (ansonsten) unbedingten Haftstrafe eingesetzt werden.

Mit unserem Titelthema »Arbeit als Strafe " und der Dokumentation eines Forschungsprojekts über neue ambulante Maßnahmen bieten wir eine umfassende Bestandsaufnahme einer sich rasch verändernden Praxis, die ohne technische Überwachung funktioniert und sich am Ziel der Resozialisierung orientiert. Hierfür, wie für alle Beiträge, wünsche ich mir Ihr Interesse und Ihnen wie immer beste Fachlektüre.

Oliver Brüchert

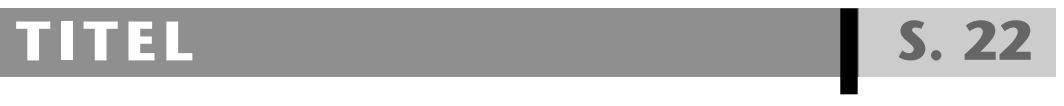

Die gemeinnützige Arbeit wird sich in absehbarer Zeit zur Hauptstrafe mausern. Damit ließen sich viele Ersatzfreiheitsstrafen und die daraus resultierenden Überbelegungen im Strafvollzug vermeiden. Doch sind noch viele Fragen offen, die sowohl die rechtlichen Rahmenbedingungen als auch die konkrete Gestaltung der Arbeitssanktion betreffen.

\section{Die Gemeinnützige Arbeit im Strafrecht}

Von Wolfgang Feuerhelm .................... 22

\section{Vermeidung von Ersatzfreiheitsstrafen durch gemeinnützige Arbeit}

Von Frieder Dünkel und Rudolf Grosser $\ldots \ldots \ldots \ldots \ldots \ldots \ldots \ldots$

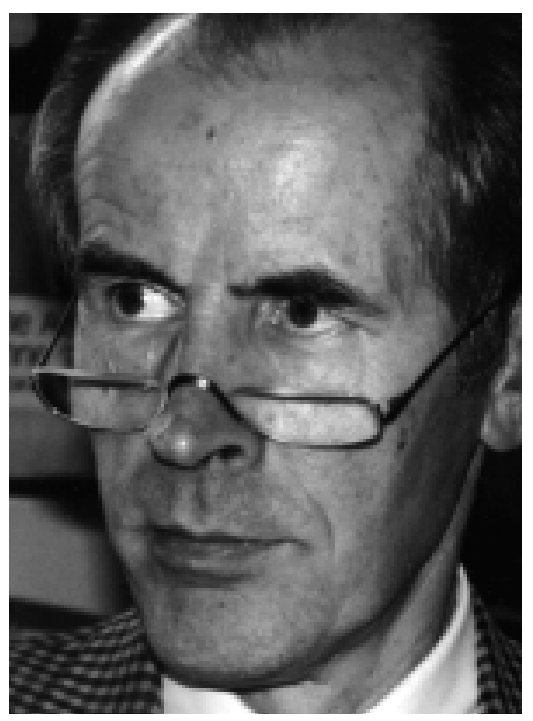

\section{FORUM \\ S. 10}

Mit der Behauptung zunehmender Jugendgewalt sorgt das Kriminologische Forschungsinstitut Niedersachsen öffentlich für Furore. Wir haben Christian Pfeiffer als Leiter des Instituts gefragt, wie haltbar die These ist.

Kriminalpolitisches Gespräch:

Jugendkriminalität als Folge sozialer Unterprivilegierung?

Klaus Boers und Manuel Eisner sprachen mit Christian Pfeiffer ........... 10

\section{THEMA}

Innere Sicherheit:

Verschwindet der Rechtsstaat?

Von Detlev Frehsee ................. 16

\section{DOKUMENTATION S. 34}

Forschungsdokumentation:

Soziale Trainingskurse und andere neue ambulante Maßnahmen

Von Frieder Dünkel, Bernd Geng und

Wolfgang Kirtsein ................... 34

\section{MAGAZIN S. 4}

Alternative Gefängnisprojekte::

Revolutionäre Rückfallquoten in Südamerika

Von Tobias Merckle ................ 4

Sicherheitspolizeigesetz in Österreich:

Variation des Wettkampfs "Hase-Igel«

Von Hermann Kuschej. .................. 6

Elektronisch überwachter Hausarrest:

\section{Strafe zu Hause?}

Von Gabriele Kawamura .............. 7

Dieser Ausgabe liegt ein Prospekt der Nomos Verlagsgesellschaft bei. Wir bitten freundlichst um Beachtung.
Als besondere Zugabe dokumentieren wir den Forschungsbericht der ersten bundesweiten Bestandsaufnahme über neue ambulante Maßnahmen im Jugendstrafrecht. Seit Anfang der neunziger Jahre haben sich diese sozialpädagogisch orientierten Ansätze zu einem nahezu flächendeckenden Angebot entwickelt. Die Befragung von Jugendrichtern, Jugendämtern und Freien Trägern ergab auch, daß ein weiterer Ausbau erwünscht und praktizierbar ist. Sonderteil ab Seite 34.

\section{RUBRIKEN}

Recht ............. 46

Terminal ............ 47

Rezensionen ........... 48

Neue Bücher . . . . . . . . . . 49

Impressum .......... 50 